Koks und Retortenkohle, bald bei Anwesenheit von Holzkohle zu bestimmen versucht hat. Am meisten hat er die letztere, unter anderen auch für eine von $\mathrm{Baur}$ und Glaessner benutzte Gleichgewichtstabelle, verwendet, also nicht graphitischen Kohlenstoff, sondern amorphen. Die Temperaturen von Baur und Glaessner sind also die Gleichgewichtstemperaturen von Eisen, Oxydul und den Gasen in Gegenwart von Holzkohle. Dass diese anders liegen müssen, als dic für die Anwesenheit von graphitischer Kohle, ist ohne weiteres klar. Der Widerspruch zwischen den Resultaten ist also hinfällig.

Wir haben mit Holzkohle Messungen nicht vorgenommen, wohl aber mit der ihr nahestehenden amorphen Zuckerkohle, es ist nun von Interesse, deren Gleichgewichtsdruck mit den Werten von Baur und Glaessner zu vergleichen. Wir fanden dort:

für x Atmosphäre:

Zuckerkohle $650^{\circ}$ Holzkohle (B. u. G.) $685^{\circ}$, für $1 / 4$ Atmosphäre:

Zuckerkohle $570^{\circ}$ Holzkohle (B. u. G.) $65^{\circ}$.

Herr Baur wird mir also wohl zugeben müssen, dass nach Lage der Verhältnisse, wenn wir von dem Viertelatmosphärendruck absehen, die Uebereinstimmung zwischen dem graphisch ermittelten und direkt beobachteten Punkte eine leidlich befriedigende ist. Dass die Holzkohle etwas andere Werte liefert als die Zuckerkohle, ist durchaus verständlich, es dürften wohl auch die einzelnen Holzkohlensorten untereinander verschieden sein, es wird dabei nicht nur die Holzart, aus der sie gewonnen wird, von Bedeutung sein, sondern auch die Temperatur, bei welcher die Verkohlung vorgenommen wurde, und die Dauer des Erhitzens. Welch grossen Finfluss diese Faktoren auf die Reaktionsfähig. keit des Kohlenstoffes haben, zeigen die Erfahrungen bei der Fabrikation des schwarzen Schiesspulvers.

Ich hoffe, durch die Aufklärungen die Fachgenossen davon überzeugt zu haben, dass unsere Versuche volles Vertrauen verdienen.
Ja, man könnte sogar noch weiter gehen, und in der angenäherten Uebereinstimmung zwischen Konstruktion und Beobachtung eine Gewähr dafür erblicken, dass wir bei den Versuchen mit den metastabilen Kohlenstoffmodifikationen die ihnen zugehörigen Gleichgewichtsdrucke tatsächlich erreicht haben. Dass wir sie erreichen konnten, liegt wieder an der Versuchsanordnung. Die Erreichung der hohen Drucke wurde durch den Umstand ermöglicht, dass die Gasentwicklung aus den festen Phasen relativ schnell, die. Spaltung des Kohlenoxydes in Gegenwart von Eisen dagegen langsam verläuft, wie wir in unserer Abhandlung auf S. $213^{6}$ bereits mitgeteilt haben. Die Geschwindigkeit der Umwandlung des Kohlenoxydes in die stabile grapbitische Modifikation hängt nun sehr von der Menge des anwesenden Metalles ab, hält man diese auf geringen Beträgen, so kann sich bequem das Gleichgewicht mit der metastabilen Modifikation ausbilden, ohne dass die Umwandlung in Graphit die Messungen storrt. Diese Störung setzt erst ein, wenn die anwesende oder gebildete Menge von metallischem Eisen gross wird.

Aus dem Mitgeteilten dürfte hervorgehen, dass die Untersuchungen über die Gleichgewichte des Systems $\mathrm{C}, \mathrm{CO}, \mathrm{CO}_{2}$ dringend einer Revision bedürfen, nicht nur im Interesse der Wissenschaft, sondern auch in dem der Technik. Um so erfreulicher ist es, dass A. Smits derartigc Messungen in Aussicht stellt. Auch wir sind damit beschäftigt, Material für diese Untersuchungen zu liefern, indem wir die im totalen Gleichgewicht mit $F e, F \varepsilon O$ und den verschiedenen Kohlenstoffmodifikationen befindlichen Gase gasanalytisch untersuchen. Es liegt ja im Interesse der guten Lösung eines Problemes, dass es von verschiedenen Seiten in verschiedener Weise in Angriff genommen wird.

Marburg, den 18. Februar 1906.

(Eingegangen: 2o. Februar)

\title{
TECHNISCHES REPERTORIUM.
}

\section{NEUERUNGEN AUF DEM GEBIET DER AKKUMULATORENTECHNIK IM JAHRE I905. \\ Von Dr. Max Roloff und Dr. Erich Siede.}

Die Akkumulatorentechnik zeigt im letzten Jahr im wesentlichen dasselbe Bild wie früher: Eine Menge Anmeldungen und Erteilungen von Patenten auf Gitter verschiedener Konstruktion, auf Verbesserungen im Einbau u. s. w. jedoch wenig für Wissenschaft und Technik interessante Ergebnisse. Im Vordergrund des Interesses steht jetzt infolge des plötzlichen Aufschwunges der Automobilindustrie, der früher selbst von den grössten Firmen oft stiefmütterlich behan- delte leichte Traktionsakkumulator. Wohl jede grössere Fabrik ist zur Zeit mit der Bearbeitung dieses Problemes beschaftigt. Auf der Automobilausstellung im Landesausstellungspark zu Berlin im April 1905 hatten die Köner Akkumulatorenwerke neben einzelnen Akkumulatoren auch einige Fahrzeuge ausgestellt, da diese Firma auch die Fahrzeugfabrikation in umfassendster Form aufgenommen bat. Die Akkumulatoren, die sie verwendet, sind ihre Typen $W$ mit etwa 
2o Wattstunden pro Kilogramm Batteriegewicht bei 150 Entladungen Lebensdauer und $W$ extra mit 30 Wattstunden bei etwa roo Entladungen für die positiven und etwas höherer Lebensdauer für die negativen Platten. Jetzt fabriziert die Firma noch die L-Type mit 34 Wattstunden Leistung pro Kilogramm Batteriegewicht. Die Akkumulatorenfabrik A.-G. Berlin-Hagen i. W. hatte zwei Elektromobilbatterieen ausgestellt, die eine für Pufferbetrieb in gemischten Automobilen, die andere für gewöhnlichen Antrieb in Elektromobilen überhaupt. Die Leistung war dieselbe wie bei Gottfried Hagen, wenn die Elemente sich auch im Einbau etwas unterschieden. Die auf der Ausstellung befindlichen Akkumulatoren der französischen Firmen hatten durchschnittlich nur eine Leistung von 20 Wattstunden pro Kilogramm Gewicht.

Die am 1. April 1905 erschienene Nummer des Centralblattes für Akkumulatoren und Elementenkunde (S. 75) brachte einen Artikel von G. Rosset-Paris, betitelt: "Der Akkumulator ,E. J. A.' mit allotropem Blei" "1). Der Verfasser behauptet hierin, dass es ihm gelungen sei, einen Aklumulator zu konstruieren, der an Leistungen alles bisher Frzielte weit übertreffe. Der Erfinder gibt an, auf Grund von genauen langjährigen Studien zu der Ueberzeugung gekommen zu sein, dass die bisher angewandten Mittel, wie Vcrminderung des Gittergewichtes, Erhöhung der Porosität der Masse, Erhöhung der Temperatur u. s. w. zur Steigerung der Leistung unzureichend wären. In einer weiteren Arbeit ${ }^{2}$ ) bespricht er eingehend seine Erfahrungen auf diesem Gebiet und stellt dann eine Formel für die Konstanten einer Zelle auf. Ist $n$ die Anzahl Ampère-Stunden pro Kilogramm Elektrode, $a$ das in Kilogramm ausgedrückte Gewicht des für I Amp.-Stunde nötigen Elektrolytes, $B+n b=$ Gewicht des Gefässes $=$ Raumbedarf von I kg Elektrode + Raumbedarf des für die Kapazität von $\mathrm{I} \mathrm{kg}$ Elektrode nötigen Elektrolyten, so erhält er $y p$ Amp.-Stunden auf I $\mathrm{kg}$ Zellengewicht durch die Gleichung:

$$
y p=\frac{n}{I+B+n(a+b)} \text {. }
$$

Die entsprechende Kurve ist cine gleichseitige Hyperbel, welche parallel zur Achse der $n$ asymptotisch verläuft. Wenn es also auch gclänge, die Kapazität auf I kg Elektrode jenseits jeder Grenze wachsen zu lassen, so würde sich nach Rosset die Kapazität auf I kg Gesamtgewicht immer weniger erhöhen.

Verfasser sucht an einer Reihe von Beobachtungen diese Behauptung $z u$ beweisen. Er ist nun zu dem Schluss gekommen, dass

I) Siehe die kurze Erwähnung des Akkumulators Z. f. Elektroch. 12, 40.

2) Centralbl. f. Akkunulatoren 1905, I 79. nur die Erhöhung der Reaktionsgeschwindigkeit durch ein anderes Mittel als Steigerung der Temperatur, und eine Verringerung der Gleichgewichtskonstante des elektrochemisch wirksamen Paares eine höhere Leistung der Zelle erzielen könnten. Rosset will nun im Jeantaudschen Laboratorium eine neue Modifikation des Bleies entdeckt haben, die diesen Forderungen vollkommen entspricht. Dieses allotrope Blei hat eine grössere Reaktionswärme, eine Eigenschaft, die im Sammler eine Erhöhung der EMK um mehr als $5 \%$ bedingt bei gleicher Konzentration der Schwefelsäure, ein geringeres spezifisches Gewicht und ein nur halb so grosses elektrochemisches Aequivalent; es ist also eine einwertig auftretende Modifikation des Bleies. Wie Rosset zu der neuen Modifikation gekommen ist, sagt er nicht.

Messungen, die an Zellen nach seinem System im Laboratoire Central d'Electricité vorgenommen wurden, ergaben auf

9,27 kg Gewicht 25 Amp. $\times 7$ Stunden $3^{6}$ Minuten $=193$ Amp.-Stunden,

I, $70 \mathrm{~kg}$ Gewicht 36 Amp; $\times 6$ Stunden 34 Minuten $=237$ Amp.-Stunden.

Dabei waren die Zellen nach Aussage des Erfinders bereits mehrere Male geladen und entladen.

Die Spannung betrug bei einer Säuredichte von $x, 31$ länger als 3 Stunden während der Entladung 2 Volt. Die Ruhespannung betrug durchschnittlich 2,28 Volt.

Durchschnittlich wurden von bereits gebrauchten Elementen 4I,2 Wattsunden pro Kilogramm geleistet.

Von anderer Seite an solchen Zellen angestellte Versuche haben Unterschiede gegenüber gewöhnlichen Bleiakkumulatoren in keiner Weise ergeben. Es ist auch von vornherein klar, dass selbst, wenn Rosset eine neue energetisch höherwertige Modifikation des Bleies gefunden hätte, diese bei der Wiederaufladung in die alte gewöhnliche Form übergehen müsste. Ueberhaupt scheint es, dass die ganze Angelegenheit sich im Sande verläuft. Bis jetzt sind diese Zellen noch nicht auf dem Markt erschienen, was bei solcher umwälzenden Neuerung sicher in kürzester Frist zu erwarten gewesen wäre. Auch sind weitere Publikationen über diese Neuerung bisher nicht erfolgt.

In Amerika wird augenblicklich für einen neuen Akkumulator viel Reklame gemacht. Es ist dies der Bijur "High Duty"-Akkumulator, der von der General Storage Battery Co. auf den Markt gebracht wird.

Derselbe ist das Ergebnis mehrjähriger Bemühungen Joseph Rijurs und des Dr. J.S. Wells. Die alte Kombination Blei-Schwefelsäure ist bei diesem Sammler zwar beibehalten, 
jedoch weicht die Platte in Plan und Konstruktion von anderen $a b^{1}$ ).

Die Ideale des Akkumulatorentechnikers: feste Struktur, Vermeidung der Deformation, vollkommene Diffusion der Säure, Festigkeit der wirksamen Masse, grosse spezifische Kapazität, Nutzeffekt u. s. w. suchte der Erfinder dadurch zu erreichen, dass er eine Platte mit kleinen unterteilten Stücken schuf, die fest an einen gut leitenden Träger angelötet waren und sich ungehindert ausdehnen konnten. Zur Herstellung der Platten musste der Erfinder eine neue Maschine und auch ein neues Formationsverfahren ersinnen. Die Platten selbst bestehen aus einer Anzahl Roste aus reinem Blei. Diese werden untereinander und mit einem starren Rahmen aus Antimonblei zusammengelötet (siehe Fig. 83).

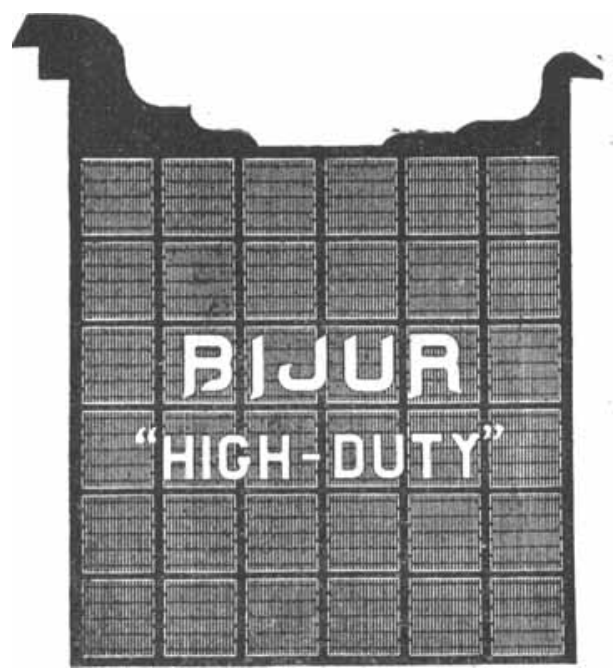

Fig. 83 .

An jedem Ende des Rostes ist ein Raum für seine Verlängerung durch Ausdehnung vorhanden. Man erhält also eine Platte mit einem nicht oxydierbaren Träger aus Hartblei, auf dem die wirksame Masse fest verankert ist. Die Roste bestehen aus einer Zahl kleiner Oeffnungen oder Zellen, die von einer Elektrodenfläche bis zur anderen offen sind. Nach der Formation füllt das Superoxyd die Oeffnungen der kleinen Zellen der Platte vollständig aus. Die Rippen nehmen dann eine schwach elliptische Form an. Geht die Oxydation weiter, so wächst die Masse längs der beiden Achsen der Killipse und lässt auch bei vollständiger Oxydation einen kleinen Schlitz frei, durch den der Elektrolyt zirkulieren kann. Das Werfen soll bei dieser Platte unmöglich sein, ebenso Sulfatation, da die leichte Diffusion der Säure jede Konzentrationserhöhung in den Poren aus-

1) Vergl, auch die Figur Z. f. Elektroch. 11, 967 . gleicht. Die Fabrikanten behaupten ferner, dass auch infolge der schnellen Säurediffusion die Elemente bei einer Spannung von 2,4 bis 2,5 Volt anstatt der 2,7 Volt, die sonst erforderlich sind, vorllig aufgeladen werden können.

Hierzu ist zu bemerken, dass jetzt überhaupt alle Grossoberflächenpositiven ohne Kern, d. h. mit durchgehenden Oeffnungen gegossen werden, und dass die Zusammensetzung grosser Platten aus kleinen Stücken schon weit früher versucht wurde, um die Krümmung zu verhindern. Wegen der erforderlichen nutzlosen Trägerflächen zwischen den Teilen hat man dieses Prinzip längst aufgegeben. Etwas Neues ist durch die Erfindung also durchaus nicht gegeben.

Eine neue Gitterkonstruktion hat sich auch William Gardiner (Engl. Patent Nr. 27905) schützen lassen. Die wirksame Masse soll am Herausfallen und Auswaschen durch den Elektrolyten beim Rütteln der Zellen gehindert werden. Ferner will er der Berührung des Elektrolyten eine möglichst grosse Fläche bieten bei möglichst geringem Metallgewicht. Er sucht dies durch ein sehr dünn ausgewalztes, dicht perforiertes Bleiblech zu erreichen, das er dann in eine Anzahl Falten legt. Die beiden Enden werden dann zusammengelegt und verschmolzen, so dass der mittlere Teil nach aussen gekrümmt bleibt. Nun schmilzt man eine Fahne an und schmilzt die benachbarten Falten an einer Anzahl von Punkten zusammen. Bei der Formation entsteht auf beiden Seiten in den Falten Superoxyd, das durch letztere sehr stark zusammengepresst wird. Auch diese Art der Plattenkonstruktion ist fruther bereits ausgeführt, aber wegen der hohen Herstellungskosten wieder verlassen worden.

Um die Stäbe der Masseträger vor der Korrosion zu bewahren, hat M. Heisser (Amerik. Pat. Nr. 794501) ein neues Gitter konstruiert. Ein Rahmen wird von einer Anzahl paralleler, horizontaler Stäbe durchsetzt, die im Querschnitt -förmige Gestalt haben. Die Ränder der Stăbe bilden scharfe Kanten. Die Stäbe sind so leicht und dünn wie möglich und werden deshalb durch mit ihnen zusammengegossene diagonale Querstäbe versteift. Die als Masseträger dienenden horizontalen Stäbe kommen also nur an ihrer scharfen Kante mit dem Elektrolyten direkt in Berührung.

Gardiner (Engl. Patent Nr. 27862) legt zwei Platten mit diagonalen Rippen aufeinander. Letztere sind nach innen zu dreikantig gestaltet. Zwischen die beiden Platten kommt die Masse, beide Träger werden dann durch einen Niet vereinigt und an den Kanten verschmolzen. Die diagonalen Rippen sind dann so angeordnet, dass sie bei der fertigen Elektrode kreuzweise zueinander verlaufen. Dadurch wird die Masse ziemlich fest gehalten. 
Eine originelle Methode zur Herstellung von Grossoberflächenplatten hat $\mathrm{Franz}$ Hobel sich patentieren lassen (D. R.-P. Nr. I60022). Der Grundgedanke besteht darin, die Rinnen eines gerippten Bleikörpers mit einer Paste von bestimmter Zusammensetzung auszufüllen (z. B. Pech, kohlensaures Natron, Schwefel), die denselben Druckwiderstand wie Blei hat, und diesen Körper durch ein enges Mundstück zu pressen. Dadurch entsteht ein dem Querschnitt nach verkleinerter Körper, der jedoch eine grössere Oberflächenentwicklung besitzt, wenn man die Paste entfernt. Wollte man einen Rippenkörper durch ein gezahntes Mundstück pressen - ohne Anwendung von Füllmaterial - , so wäre ein bedeutend grösserer Druck notwendig, als bei diesem Verfahren. Ausserdem sind genügend starke Zähne schwer herzustellen. Um Akkumulatorenplatten herzustellen, geht der Erfinder von einem grösseren Pressblock aus, der bereits mit relativ groben, horizontal und vertikal verlaufenden Rippen versehen ist. Die Rinnen werden dann mit Füllmasse ausgefüllt und der Block wird durch ein enges Mundstück gepresst. So entsteht schliesslich ein mehrere Millimeter breites und mehrere Centimeter langes Band.

Die Vertikalrippen sind feine Lamellen geworden, die Querrippen bilden massive Zwischenstücke. Aus letzteren werden dann die Fahnen ausgestanzt. So hübsch auch dieses Verfahren ist, kann man doch wohl kaum der Behauptung des Erfinders glauben, dass es den Vorzug der Billigkeit hat. Grössere Platten nach diesem Verfahren herzustellen ist nicht gut möglich. Die vom Erfinder besonders betonte leichte Vermehrung der Entwicklung (bis auf das $5^{\circ}$ fache) der linearen Oberfläche hat auch wenig $Z$ weck, da man über eine neunfache Entwicklung praktisch nicht hinausgehen kann, indem die Säurediffusion in den engen Spalten behindert wird.

E. A.Sperry (Amerik. Pat. Nr. 773685) stellt Bleikarbonat durch Fällen oder elektrolytisch her und rosstet es, so dass ein Gemisch von ro Teilen $\mathrm{PbO}$ und 12 Teilen $\mathrm{Pb}_{3} \mathrm{O}_{4}$ entsteht. Damit pastiert er Gitter direkt oder besser noch mit einem Zusatz von Ammoniumsulfat. Dann löst er letzteres mit Wasser heraus, wodurch die Masse erhärtet und die Zuleitungsporen für den Elektrolyten freigelegt werden.

Von Neuerungen auf dem Gebiete der For- mation ist in erster Linie das Patent von Dr. Diamant zu nennen (D. R.-P. Nr. I57 I95). Während Diamant früher der Formierflüssigkeit unmittelbar Methyl- und Aethylalkohol oder deren Oxydationsprodukte Ameisensäure und Essigsäure zusetzten, behandelt er jetzt diese Verbindungen zuerst mit konzentrierter Schwefelsäure, bezw. deren Anhydrid und erhält so Sulfo-, resp. Oxysulfosäurederivate des Methans. Diese Verbindungen werden der Formierflüssigkeit zugesetzt, wie der Erfinder behauptet, mit gutem Erfolge. Ausserdem sind diese Zusătze leicht aus den formierten Platten vollständig zu entfernen. Da nur geringe Mengen notwendig sind, hat auch das Verfahren den Vorzug grosser Billigkeit. - Henry Leitner (Engl. Pat. Nr. 6792) schlägt vor, die Platten für die Formation durch Behandlung im Elektrolyten von verdünnten Alkalisulfatlösungen, denen Spuren bleilösender Stoffe zugefügt sind, vorzubereiten. Der Elektrolyt soll $2 \%$ Natriumsulfat und auch etwa $2 \%$ Chloride enthalten. Bessere Resultate (Engl. Pat. Nr. I4 128) hat er jedoch noch erhalten, wenn er statt der Salze ihre freien Säuren in entsprechenden Gewichtsmengen verwendete. Bei dieser Behandlung ist darauf $\mathrm{zu}$ achten, dass die Temperatur $30^{0}$ nicht übersteigt. Danach müssen die Platten gut ausgewässert werden, worauf man sie in Schwefelsäure weiter formiert. Sperry (Amerik. Pat. Nr. 79I 795) überzieht Masseplatten mit einer schützenden Hülle von Pyroxylin, um das Ausfallen der Masse zu hindern. Er hat Hartbleigitter mit grossen Maschen, in die er die mit Pyroxylingewebe überzogenen Massekuchen einlegt. Dadurch soll die Masse auch weniger empfindlich gegen Stösse sein. Achille Meygret (Amerik. Pat. Nr. 776 192) taucht Masseplatten in eine Lösung von Cellulosetetraacetat allein oder im Gemisch mit Butyrat. Der Ueberzug ist dann elastisch und widersteht dem Angriffe des Elektrolyten; er wird dann noch mit feinen Nadeln perforiert.

Ein zu straffes Anspannen des Ueberzuges sucht er dadurch zu verhindern, dass er die Platten vorher in eine dick flüssige Lösung von Saccharose taucht. Der Elektrolyt löst dann die Saccharose wieder heraus und macht die Masse porös, so dass die Gefahr einer Zerstörung der Tetracetathülle beim Quellen der Masse vermieden wird.

\section{DEUTSCHE PATENTNACHRICHTEN}

für die chemische, elektrochemische und elektrometallurgische Technik.

\section{Deutschland.}

Patentanmeldungen.

Bekannt gemacht im Reichsanzeiger rom I. März 1906:

12k. W. 23822. Verfahren zur Gewinnung von Ammoniak aus dem Stickstoff der Luft durch Ueberleiten von Luft und Wasserdampf über erhitzten Torf; Zus. z. Anm. W. 2r 257. H. C. Woltereck, London. 29. 4.05 .

I2p. H. 35438 . Verfahren zur Darstellung von Theobrominnatrium-Natriumformiat. F. Hof $\mathrm{m}$ an $\mathrm{n}-\mathrm{La}$ Roche \& Cie., Basel. 30. 5. 05 . 